\title{
MONGE-AMPÈRE EQUATIONS AND SURFACES WITH NEGATIVE GAUSSIAN CURVATURE
}

\author{
MIKIO TSUJI \\ Department of Mathematics, Kyoto Sangyo University \\ Kamigamo, Kita-ku, Kyoto 603, Japan \\ E-mail:mtsuji@cc.kyoto-su.ac.jp
}

\begin{abstract}
In [24], we studied the singularities of solutions of Monge-Ampère equations of hyperbolic type. Then we saw that the singularities of solutions do not coincide with the singularities of solution surfaces. In this note we first study the singularities of solution surfaces. Next, as the applications, we consider the singularities of surfaces with negative Gaussian curvature. Our problems are as follows: 1) What kinds of singularities may appear?, and 2) How can we extend the surfaces beyond the singularities?
\end{abstract}

1. Introduction. In our previous work [24], we studied the singularities of solutions of real Monge-Ampère equations of hyperbolic type as follows: Let $z=z(x, y)$ be an unknown function defined for $(x, y) \in \mathbf{R}^{2}$, then the equation is written as

$$
F(x, y, z, p, q, r, s, t)=A r+B s+C t+D\left(r t-s^{2}\right)-E=0
$$

where $p=\partial z / \partial x, q=\partial z / \partial y, r=\partial^{2} z / \partial x^{2}, s=\partial^{2} z / \partial x \partial y$, and $t=\partial^{2} z / \partial y^{2}$. Here we assume that $A, B, C, D$ and $E$ are real smooth functions of $(x, y, z, p, q)$. As we can see that the singularities of solution $z=z(x, y)$ of (1.1) do not coincide with the singularities of solution surface $\{(x, y, z) ; z=z(x, y)\}$, we will first study the singularities of solution surfaces of (1.1). Next, as the application, we consider the singularities of surfaces with negative Gaussian curvature. Concerning the second subject, we will give some comments. It is well known that a surface in $\mathbf{R}^{3}$ with constant negative Gaussian curvature has singular points. (For example, see D. Hilbert [8] and E. Holmgren [9].) But we do not know what kinds of singularities may appear. Moreover we would like to extend the solution surface beyond the singularities. Our problems are to answer to the above two questions. As it seems to us that we do not have any result on these problems, we think that, though we assume a little strong conditions, this is one step to construct the global theory on hyperbolic surfaces. We cannot treat, by the method developed in this note,

1991 Mathematics Subject Classification: Primary 35L70; Secondary 53C21, 58C27, 58G17. The paper is in final form and no version of it will be published elsewhere. 
the surface whose Gaussian curvature is negative constant, though we will give some comments on this subject in the last section. In this note we will give only the sketch of our results. The detailed proofs will be given in the forthcoming paper.

2. Characteristic method and intermediate integrals. The aim of this talk is to construct the singularities of the solution surfaces in the case where equation (1.1) is hyperbolic. For our aim, we have to represent the solutions explicitly. To do so, we apply the characteristic method developed principally by D. Darboux and E. Goursat ([3], [5], [6]). As it seems to us that the method is not familiar today, we will briefly explain it in Section 2 "from our point of view". But this method depends on a property that the dimension of space is two. The main idea of Darboux and Goursat is how to reduce the solvability of (1.1) to the integration of first order partial differential equations, though their theory is local. But, as their method is constructive, it is very useful for our purpose. Let

$$
\Gamma:(x, y, z, p, q)=(x(\alpha), y(\alpha), z(\alpha), p(\alpha), q(\alpha)), \quad \alpha \in \mathbf{R}^{1},
$$

be a smooth curve in $\mathbf{R}^{5}$, and suppose that it satisfies the following "strip condition":

$$
\frac{d z}{d \alpha}(\alpha)=p(\alpha) \frac{d x}{d \alpha}(\alpha)+q(\alpha) \frac{d y}{d \alpha}(\alpha) .
$$

As a "characteristic strip" means that one cannot determine the values of the second order derivatives of solution along the strip $\Gamma$, we have the following

Definition 2.1. A curve $\Gamma$ in $\mathbf{R}^{5}=\{(x, y, z, p, q)\}$ is a "characteristic strip" if it satisfies (2.1) and

$$
\operatorname{det}\left[\begin{array}{ccc}
F_{r} & F_{s} & F_{t} \\
\dot{x} & \dot{y} & 0 \\
0 & \dot{x} & \dot{y}
\end{array}\right]=F_{t} \dot{x}^{2}-F_{s} \dot{x} \dot{y}+F_{r} \dot{y}^{2}=0
$$

where $F_{t}=\partial F / \partial t, F_{s}=\partial F / \partial s, F_{r}=\partial F / \partial r, \dot{x}=d x / d \alpha$ and $\dot{y}=d y / d \alpha$.

Denote the discriminant of $(2.2)$ by $\Delta$, then

$$
\Delta=F_{s}^{2}-4 F_{r} F_{t}=B^{2}-4(A C+D E) .
$$

If $\Delta<0$, equation (1.1) is called to be elliptic. If $\Delta>0$, it is hyperbolic. In this note, we will treat the equations of hyperbolic type. More precisely, we assume $\Delta \geq 0$ and also $D \neq 0$. Let $\lambda_{1}$ and $\lambda_{2}$ be the solutions of $\lambda^{2}+B \lambda+(A C+D E)=0$, then the characteristic strip satisfies the following equations:

$$
\left\{\begin{array}{c}
d z-p d x-q d y=0 \\
D d p+C d x+\lambda_{1} d y=0 \\
D d q+\lambda_{2} d x+A d y=0
\end{array}\right.
$$

or

$$
\left\{\begin{array}{c}
d z-p d x-q d y=0 \\
D d p+C d x+\lambda_{2} d y=0 \\
D d q+\lambda_{1} d x+A d y=0 .
\end{array}\right.
$$


Let $\omega_{0}=d z-p d x-q d y, \omega_{1}=D d p+C d x+\lambda_{1} d y$ and $\omega_{2}=D d q+\lambda_{2} d x+A d y$. Take an exterior product of $\omega_{1}$ and $\omega_{2}$, and substitute into their product the contact relations $\omega_{0}=0, d p=r d x+s d y$ and $d q=s d x+r d y$. Then we get

$$
\omega_{1} \wedge \omega_{2}=D\left\{A r+B s+C t+D\left(r t-s^{2}\right)-E\right\} d x \wedge d y
$$

In a space whose dimension is greater than two, the decomposition as above is not possible in general. Here we introduce the notion of "first integral".

Definition 2.2. A function $V=V(x, y, z, p, q)$ is called the first integral of $\left\{\omega_{0}, \omega_{1}, \omega_{2}\right\}$ if $d V \equiv 0 \bmod \left\{\omega_{0}, \omega_{1}, \omega_{2}\right\}$.

Rem ark. We can easily see that a function $V=V(x, y, z, p, q)$ is the first integral of (2.3) (or (2.4)) if it is constant on any solution of (2.3) (or of (2.4), respectively).

G. Darboux [3] and E. Goursat [5], especially in [5], had considered equations (1.1) under the assumption that (2.3), or (2.4), has at least two independent first integrals. We denote them by $u$ and $v$. Then we get the following

Proposition 2.3. Assume that $\lambda_{1} \neq \lambda_{2}$, and that (2.3), or (2.4), has two independent first integrals $\{u, v\}$. Then we can prove that there exists a function $k=k(x, y, z, p, q) \neq 0$ satisfying

$$
d u \wedge d v=k \omega_{1} \wedge \omega_{2}=k D\left\{A r+B s+C t+D\left(r t-s^{2}\right)-E\right\} d x \wedge d y .
$$

If equation (1.1) is written as (2.6), it would be obvious that (2.3), or (2.4), has two independent first integrals $\{u, v\}$. Therefore the representation (2.6) means the characterization of "Monge-Ampère equations of Darboux-Goursat type".

Let $\{u, v\}$ be two independent first integrals of (2.3). For any function $g$ of two variables whose gradient does not vanish, $g(u, v)=0$ is called an intermediate integral of (1.1). Let $C_{0}$ be an initial strip defined in $\mathbf{R}^{5}=\{(x, y, z, p, q)\}$. If the strip $C_{0}$ is not characteristic, we can find an intermediate integral $g(u, v)$ which vanishes on $C_{0}$. Here we put $g(u, v)=f(x, y, z, p, q)$. The Cauchy problem for (1.1) satisfying the initial condition $C_{0}$ is to look for a solution $z=z(x, y)$ of (1.1) which contains the strip $C_{0}$, i.e., the two-dimensional surface $\{(x, y, z(x, y), \partial z / \partial x(x, y), \partial z / \partial y(x, y))\}$ in $\mathbf{R}^{5}$ containing $C_{0}$. The representation (2.6) assures that, as $d u \wedge d v=0$ on a surface $g(u, v)=0$, a smooth solution of $f(x, y, z, \partial z / \partial x, \partial z / \partial y)=0$ satisfies equation (1.1). Therefore we get the following

Theorem 2.4 ([3], [5]). Assume that the initial strip $C_{0}$ is not characteristic. Then a function $z=z(x, y)$ is a solution of the Cauchy problem for (1.1) with the initial condition $C_{0}$ if and only if it is a solution of $f(x, y, z, \partial z / \partial x, \partial z / \partial y)=0$ satisfying the same initial condition $C_{0}$.

3. How to construct examples. When a partial differential equation of first order

$$
f(x, y, z, p, q)=0
$$

is given, we will construct Monge-Ampère equation which accepts equation (3.1) as its intermediate integral. Assume $(\operatorname{grad} f) \neq 0$. Then we can locally find a function $g=$ 
$g(x, y, z, p, q)$ satisfying

$$
\operatorname{rank}\left(\begin{array}{l}
\operatorname{grad} f \\
\operatorname{grad} g
\end{array}\right)=2 .
$$

Here we take a product $d f \wedge d g$ and substitute there the contact relations $\omega_{0}=0, d p=$ $r d x+s d y$ and $d q=s d x+r d y$. Then we get

$$
d f \wedge d g=F(x, y, z, p, q, r, s, t) d x \wedge d y .
$$

This representation teaches us that the equation $F=0$ has two independent first integrals $f$ and $g$. At today's point, we do not have the proof of global existence of the function $g=g(x, y, z, p, q)$ satisfying the property (3.2). Therefore the equation $F=$ $F(x, y, z, p, q, r, s, t)$ has the meaning only in a domain where the function $g(x, y, z, p, q)$ is defined. But we can construct the equation $F(x, y, z, p, q, r, s, t)=0$ in the large for equations of certain types as follows.

EXAmple 1. Assume that $f$ is of Hamilton-Jacobi type, i.e., $f=p+h(x, y, z, q)$. Choosing the function $g$ as $g=q+k(x, y, z)$, we construct the equation $F=0$ by

$$
\begin{aligned}
d f \wedge d g & =\left\{\left(r t-s^{2}\right)+A r+B s+C t-E\right\} d x \wedge d y \\
& =F(x, y, z, p, q, r, s, t) d x \wedge d y
\end{aligned}
$$

where $A, B, C$ and $E$ are certain functions of $(x, y, z, p, q)$.

ExAmple 2. Assume that $f$ is quasi-linear, i.e., $f=a p+b q+c$ where $a, b$ and $c$ are real smooth functions of $(x, y, z)$ and $(a, b) \neq(0,0)$. Choose $g=-b p+a q+c^{\prime}$ where $c^{\prime}$ is an arbitrary function of $(x, y, z)$. Then the equation $F=0$ is obtained by

$$
\begin{aligned}
d f \wedge d g & =\left\{\left(a^{2}+b^{2}\right)\left(r t-s^{2}\right)+A r+B s+C t-E\right\} d x \wedge d y \\
& =F(x, y, z, p, q, r, s, t) d x \wedge d y
\end{aligned}
$$

where $A, B, C$ and $E$ are functions of $(x, y, z, p, q)$.

EXAMPLE 3. Any non-characteristic Cauchy problem for first order partial differential equations can be locally reduced to the following form:

$$
\begin{gathered}
\frac{\partial u}{\partial x}+f\left(x, y, u, \frac{\partial u}{\partial y}\right)=0 \quad \text { in } \quad\left\{(x, y) ; x>0, y \in \mathbf{R}^{1}\right\} \\
u(0, y)=\varphi(y) \quad \text { on } \quad\left\{(0, y) ; x=0, y \in \mathbf{R}^{1}\right\} .
\end{gathered}
$$

By Example 1, we can construct Monge-Ampère equation which accepts equation (3.6) as the intermediate integral. Following the Cauchy problem (3.6) - (3.7), we define the initial strip $C_{0}$ by

$$
C_{0}:(x, y, z, p, q)=\left(0, \alpha, \varphi(\alpha),-f\left(0, \alpha, \varphi(\alpha), \varphi^{\prime}(\alpha)\right), \varphi^{\prime}(\alpha)\right), \alpha \in \mathbf{R}^{1} .
$$

Then we can easily see that the Cauchy problem for Monge-Ampère equation with the initial strip $C_{0}$ has the Cauchy problem (3.6) - (3.7) as its intermediate integral.

4. Solution surfaces of Monge-Ampère equations. In this section we will study the singularities of solution surfaces of Monge-Ampère equations. Here it would be better to make clear the meaning of "singularity" of surfaces, though we may write very elementary facts. 
Definition 4.1. A point $\left(x^{0}, y^{0}, z^{0}\right)$ is called to be a singularity of a solution $z=$ $z(x, y)$ of (1.1) if and only if $z^{0}=z\left(x^{0}, y^{0}\right)$ and $z(x, y) \notin C^{2}$ in a neighbourhood of $\left(x^{0}, y^{0}\right)$.

Definition 4.2. Let $S$ be a surface in $\mathbf{R}^{3} . S$ is regular at a point $\left(x^{0}, y^{0}, z^{0}\right)$ if we can choose parameters $(\alpha, \beta) \in \mathbf{R}^{2}$ as follows: $x=x(\alpha, \beta), y=y(\alpha, \beta)$ and $z=z(\alpha, \beta)$ satisfy the two conditions:

(i) $\left(x\left(\alpha^{0}, \beta^{0}\right), y\left(\alpha^{0}, \beta^{0}\right), z\left(\alpha^{0}, \beta^{0}\right)\right)=\left(x^{0}, y^{0}, z^{0}\right)$, and $x=x(\alpha, \beta), y=y(\alpha, \beta)$ and $z=z(\alpha, \beta)$ are of class $C^{1}$ in a neighbourhood of $\left(\alpha^{0}, \beta^{0}\right)$,

$$
\operatorname{rank}\left(\begin{array}{ccc}
\frac{\partial x}{\partial \alpha} & \frac{\partial y}{\partial \alpha} & \frac{\partial z}{\partial \alpha} \\
\frac{\partial x}{\partial \beta} & \frac{\partial y}{\partial \beta} & \frac{\partial z}{\partial \beta}
\end{array}\right)=2
$$

Definition 4.3. A point $\left(x^{0}, y^{0}, z^{0}\right)$ is called to be a singularity of a solution surface $S$ if and only if $S$ is not regular at the point $\left(x^{0}, y^{0}, z^{0}\right)$.

First we will show that the singularities of solutions do not generally coincide with those of solution surfaces.

THEOREM 4.4. Assume the following two conditions:

I) (2.3), or (2.4), has two independent first integrals, and

II) The intermediate integral is quasi-linear with respect to $(p, q)$.

Then the smooth solution surface of (1.1) exists in the large, though the solution $z=z(x, y)$ of (1.1) may have singularities.

Sketch of the proof. By the assumption, we can write the Cauchy problem for the intermediate integral $f=f(x, y, z, p, q)$ as

$$
\left\{\begin{array}{c}
f=f(x, y, z, p, q)=a p+b q-c=0 \\
C_{0}:(x, y, z, p, q)=\left(x_{0}(\alpha), y_{0}(\alpha), z_{0}(\alpha), p_{0}(\alpha), q_{0}(\alpha)\right),
\end{array}\right.
$$

where $a, b$ and $c$ are smooth functions of $(x, y, z)$. The curve $C_{0}$ is the initial condition. Then the characteristic equations for (4.1) are

$$
\frac{\partial x}{\partial \beta}=a, \frac{\partial y}{\partial \beta}=b, \frac{\partial z}{\partial \beta}=c ; \quad x(0)=x_{0}(\alpha), y(0)=y_{0}(\alpha), z(0)=z_{0}(\alpha) .
$$

We denote the solutions of (4.2) by $x=x(\alpha, \beta), y=y(\alpha, \beta)$ and $z=z(\alpha, \beta)$. If the initial condition $C_{0}$ is not characteristic, we can prove that

$$
\operatorname{rank}\left(\begin{array}{lll}
\frac{\partial x}{\partial \alpha} & \frac{\partial y}{\partial \alpha} & \frac{\partial z}{\partial \alpha} \\
\frac{\partial x}{\partial \beta} & \frac{\partial y}{\partial \beta} & \frac{\partial z}{\partial \beta}
\end{array}\right)=2 \text { for any }(\alpha, \beta) \in \mathbf{R}^{2} .
$$

Therefore, though the solution $z=z(x, y)$ has singularities at the points where the Jacobian $D(x, y) / D(\alpha, \beta)=0$, the solution surface is regular even at these points.

Next we consider the case where the singularities may appear on the solution surfaces of (1.1). 
THEOREM 4.5. Assume the following two conditions:

I) (2.3), or (2.4), has two independent first integrals, and

II) The intermediate integral is of Hamilton-Jacobi type.

Then $z=z(x, y)$ is singular at a point $\left(x^{0}, y^{0}\right)$ if and only if the solution surface $\{(x, y, z) ; z=z(x, y)\}$ is not regular at a point $\left(x^{0}, y^{0}, z^{0}\right)$ where $z^{0}=z\left(x^{0}, y^{0}\right)$.

Sketch of the proof. By the assumption on the intermediate integral $f=$ $f(x, y, z, p, q)$, we can write the Cauchy problem as follows:

$$
\left\{\begin{array}{c}
f(x, y, z, \partial z / \partial x, \partial z / \partial y)=\partial z / \partial x+h(x, y, z, \partial z / \partial y)=0 \\
z(x(\alpha), y(\alpha))=z(\alpha), \quad(\partial z / \partial x)(x(\alpha), y(\alpha))=p(\alpha), \\
(\partial z / \partial y)(x(\alpha), y(\alpha))=q(\alpha) .
\end{array}\right.
$$

The characteristic differential equations for (4.3) are written as follows:

$$
\left\{\begin{array}{l}
\frac{d x}{d \beta}=\frac{\partial f}{\partial p}(x, y, z, p, q), \quad \frac{d y}{d \beta}=\frac{\partial f}{\partial q}(x, y, z, p, q) \\
\frac{d z}{d \beta}=p \frac{\partial f}{\partial p}(x, y, z, p, q)+q \frac{\partial f}{\partial q}(x, y, z, p, q) \\
\frac{d p}{d \beta}=-\frac{\partial f}{\partial x}(x, y, z, p, q)-p \frac{\partial f}{\partial z}(x, y, z, p, q) \\
\frac{d q}{d \beta}=-\frac{\partial f}{\partial y}(x, y, z, p, q)-q \frac{\partial f}{\partial z}(x, y, z, p, q),
\end{array}\right.
$$

$$
x(0)=x(\alpha), y(0)=y(\alpha), z(0)=z(\alpha), p(0)=p(\alpha), q(0)=q(\alpha), \quad \alpha \in \mathbf{R}^{1} .
$$

We denote the solutions of $(4.4)-(4.5)$ by $x=x(\alpha, \beta), y=y(\alpha, \beta), z=z(\alpha, \beta)$, $p=p(\alpha, \beta)$ and $q=q(\alpha, \beta)$. As $\omega_{0}=0$ on the solution surface, we have

$$
\operatorname{rank}\left(\begin{array}{ccc}
\frac{\partial x}{\partial \alpha} & \frac{\partial y}{\partial \alpha} & \frac{\partial z}{\partial \alpha} \\
\frac{\partial x}{\partial \beta} & \frac{\partial y}{\partial \beta} & \frac{\partial z}{\partial \beta}
\end{array}\right)=\operatorname{rank}\left(\begin{array}{cc}
\frac{\partial x}{\partial \alpha} & \frac{\partial y}{\partial \alpha} \\
\frac{\partial x}{\partial \beta} & \frac{\partial y}{\partial \beta}
\end{array}\right) \quad \text { for any }(\alpha, \beta) \in \mathbf{R}^{2}
$$

It is well known that the solution $z=z(x, y)$ has singularities at the points where the Jacobian $D(x, y) / D(\alpha, \beta)=0$. Then equation (4.6) means that the solution surface is also not regular at the points where the Jacobian vanishes. From these ideas, we can arrive at the above conclusion.

Concerning the solution surface $S=\{(x, y, z) ; z=z(x, y)\}$, the problems which we are interested in are as follows: I) What kinds of singularities may appear?, and II) Can we extend the solution surface beyond the singularities? For the problem II), we have two directions. After the appearance of singularities, the solution $z=z(x, y)$ takes in general several values. One way is to introduce a physical point of view. Then a solution must be single-valued. For this aim, we cut off some parts of solution so that it could become a single-valued weak or generalized solution satisfying the entropy condition for equations of conservation law or the semi-concavity condition for Hamilton-Jacobi equations. By this procedure, the singularities may appear in the solution. See [22], [23], [10], [11], [13], [19] and [20]. Another way is to consider the above problem from geometric point of view. 
Then we must accept multi-valued solutions. As Monge-Ampère equations appear often in geometric problems, we should take here the second approach. This means that, without cutting off some part of solution surfaces, we should accept the whole part of solution surfaces and consider the singularities of surfaces in the meaning of Definition 4.3.

To state our results, we introduce a smooth mapping $H$ from $\mathbf{R}^{2}$ to $\mathbf{R}^{2}$ defined by

$$
H(\alpha, \beta)=(x(\alpha, \beta), y(\alpha, \beta)), \quad(\alpha, \beta) \in \mathbf{R}^{2},
$$

where $x(\alpha, \beta)$ and $y(\alpha, \beta)$ are the solutions of (4.4) - (4.5). Let us write $\Sigma=\{(\alpha, \beta) \in$ $\left.\mathbf{R}^{2} ; D(x, y) / D(\alpha, \beta)=0\right\}$ and $H(\Sigma)=\Gamma$. Then we get the following

THEOREM 4.6. Let the assumptions of Theorem 4.5 be satisfied and the singularities of the mapping $H$ be fold and cusp points only. Then the curve $\Gamma$ becomes piecewise smooth and the solution surface has the singularities along $\Gamma$. Moreover we can uniquely extend the solution surface beyond the singularities in the space of $C^{1}$-functions which are of class $C^{2}$ except on piecewise smooth curves.

The canonical forms of cusp and fold points are obtained by H. Whitney [25]. The uniqueness of the extension of solution surfaces beyond the singularities follows from Theorem 4.6 in [23].

R e m a rk. Let us explain the meaning of "Hamilton-Jacobi type" used in Theorem 4.5 and 4.6. In [22], we have studied the differences between Hamilton-Jacobi equations and equations of conservation law, under the assumption that $f(x, y, z, p, q)$ is smooth. Our conclusion is that the most characteristic property of Hamilton-Jacobi equations is the global solvability of the Cauchy problem for (4.4). On the other hand, if $f=0$ is quasi-linear, the solutions $p(\alpha, \beta)$ and $q(\alpha, \beta)$ tend to infinity when the Jacobian $D(x, y) / D(\alpha, \beta)$ vanishes. Therefore, in the above theorem, "Hamilton-Jacobi type" means the global solvability of the Cauchy problem for (4.4). Recently S. Izumiya [11] has given the geometric characterization of Hamilton-Jacobi equations and quasi-linear partial differential equations of first order.

5. Surfaces with negative Gaussian curvature. Let $\kappa$ be the Gaussian curvature of the surface $z=z(x, y)$, then $z=z(x, y)$ satisfies the following equation:

$$
r t-s^{2}=\kappa\left(1+p^{2}+q^{2}\right)^{2} .
$$

We use the same notations as in Section 1. Here we are interested in the hyperbolic case, i.e., we assume $\kappa<0$. Let us recall the classical theorem due to D. Hilbert as follows:

ThEOREM 5.1 ([8]). A surface $S$ in $\mathbf{R}^{3}$ with constant negative Gaussian curvature has singular points.

Therefore, when we may extend the classical solution of (5.1), the singularities may appear in general. But, if the Gaussian curvature is not strictly negative, there exists a surface in the large whose Gaussian curvature is negative.

ExAmple. Assume the Gaussian curvature $\kappa=-1 /\left(1+p^{2}+q^{2}\right)^{2}$. Then equation (5.1) is written as

$$
r t-s^{2}=-1 .
$$


The first integrals of (5.2) are given by $\{x+q, y-p\}$. Let the initial strip $C_{0}$ be

$$
C_{0}:(x, y, z, p, q)=\left(0, \alpha, 1 / 2 \alpha^{2}, 0, \alpha\right), \quad \alpha \in \mathbf{R}^{1} .
$$

Then the intermediate integral $g=g(x, y, z, p, q)$ for the Cauchy problem $(5.2)-(5.3)$ is given by $g=p+q+x-y$. Therefore the solution of (5.2) $-(5.3)$ is written as $z=-1 / 2 x^{2}+1 / 2 y^{2}$. This means that there exists a smooth surface in the large whose Gaussian curvature is equal to $-1 /\left(1+p^{2}+q^{2}\right)^{2}$.

As the generalization of Hilbert's theorem [8], N. V. Efimov proved the following

THEOREM 5.2 (Efimov [4]). No surface can be immersed in $\mathbf{R}^{3}$ so as to be complete in the induced Riemannian metric, with strictly negative Gaussian curvature.

We write $\lambda_{1}=\left(1+p^{2}+q^{2}\right)(-\kappa)^{1 / 2}, \lambda_{2}=-\lambda_{1}, \omega_{1}=d p+\lambda_{1} d y$ and $\omega_{2}=d q+\lambda_{2} d x$. Then we have on $\left\{\omega_{0}=d z-p d x-q d y=0, d p=r d x+s d y, d q=s d x+r d y\right\}$

$$
\omega_{1} \wedge \omega_{2}=\left\{\left(r t-s^{2}\right)-\kappa\left(1+p^{2}+q^{2}\right)^{2}\right\} d x \wedge d y .
$$

Therefore equation (5.1) is obtained by the product of $\omega_{1}$ and $\omega_{2}$. Then we get

TheOrem 5.3. Assume that the Gaussian curvature $\kappa$ is a function of $(p, q)$ and strictly negative, then the system of one forms $\left\{\omega_{0}, \omega_{1}, \omega_{2}\right\}$ does not have two independent first integrals.

Therefore we cannot apply our preceding method to solve (5.1). Then our problem is how we can get a family of characteristic strips. We can obtain it by solving the following system of first order partial differential equations:

$$
\left\{\begin{array}{l}
\frac{\partial p}{\partial \alpha}+\lambda_{1} \frac{\partial y}{\partial \alpha}=0 \\
\frac{\partial q}{\partial \alpha}-\lambda_{1} \frac{\partial x}{\partial \alpha}=0 \\
\frac{\partial p}{\partial \beta}-\lambda_{1} \frac{\partial y}{\partial \beta}=0 \\
\frac{\partial q}{\partial \beta}+\lambda_{1} \frac{\partial x}{\partial \beta}=0
\end{array}\right.
$$

The local solvability of (5.4) is already proved by H. Lewy [14] and J. Hadamard [7]. But, to develop the global theory, we would have to consider the global behaviour of the solutions of (5.4).

Finally we would like to give some comments on M. Kossowski [13]. He constructed local solutions of (1.1) by the method which is almost similar to the characteristic method. Then the biggest problem is how to get the family of characteristic strips. In [23], he could obtain it by solving certain system of equations which is numbered as (7) in [13]. His equation (7) in [13] is corresponding to (5.4) in our case. As he assumed the analyticity on equations (1.1), he could solve it by Cauchy-Kowalewski theorem. As we consider (1.1) in $C^{\infty}$-space, we need the condition of hyperbolicity on (1.1). 


\section{References}

[1] M.-H. Amsler, Des surfaces à courbure constante négative dans l'espace à trois dimensions et de leurs singularités, Math. Ann. 130 (1955), 234-256.

[2] R. Courant and D. Hilbert, Method of Mathematical Physics, vol. 2, Interscience, New York, 1962.

[3] G. Darboux, Leçon sur la théorie générale des surfaces, tome 3, Gauthier-Villars, Paris, 1894.

[4] N. V. Efimov, Generation of singularities on surfaces of negative curvature, Mat. Sb. 64 (1964), 286-320.

[5] E. Goursat, Leçons sur l'intégration des équations aux dérivées partielles du second ordre, tome 1, Hermann, Paris, 1896.

[6] E. Goursat, Cours d'analyse mathématique, tome 3, Gauthier-Villars, Paris, 1927.

[7] J. Hadamard, Le problème de Cauchy et les équations aux dérivées partielles linéaires hyperboliques, Hermann, Paris, 1932.

[8] D. Hilbert, Über Flächen von constanter Gausscher Krümmung, Trans. Amer. Math. Soc. 2 (1901), 87-99.

[9] E. Holmgen, Sur les surfaces à courbure constante négative, C. R. Acad. Sci. Paris 134 (1902), 740-743.

[10] S. Izumiya, Geometric singularities for Hamilton-Jacobi equation, Adv. Stud. Pure Math. 22 (1993), 89-100.

[11] S. Izumiya, Characteristic vector fields for first order partial differential equations, preprint.

[12] S. Izumiya and G. T. Kossioris, Semi-local classification of geometric singuarities for Hamilton-Jacobi equations, J. Differential Equations 118 (1995), 166-193.

[13] M. Kossowski, Local existence of multivalued solutions to analytic symplectic MongeAmpère equations, Indiana Univ. Math. J. 40 (1991), 123-148.

[14] H. Lewy, Über das Anfangswertproblem einer hyperbolischen nichtlinearen partiellen Differentialgleichung zweiter Ordnung mit zwei unabhängigen Veränderlichen, Math. Ann. 98 (1928), 179-191.

[15] H. Lewy, A priori limitations for solutions of Monge-Ampère equations I, II, Trans. Amer. Math. Soc. 37 (1934), 417-434; 41 (1937), 365-374.

[16] V. V. Lychagin, Contact geometry and non-linear second order differential equations, Russian Math. Surveys 34 (1979), 149-180.

[17] T. K. Milnor, Efimov's theorem about complete immersed surfaces of negative curvature, Adv. Math. 8 (1972), 474-543.

[18] T. Morimoto, La géométrie des équations de Monge-Ampère, C. R. Acad. Sci. Paris Sér. I Math. 289 (1979), 25-28.

[19] S. Nakane, Formation of singularities for Hamilton-Jacobi equations in several space variables, J. Math. Soc. Japan 43 (1991), 89-100.

[20] S. Nakane, Formation of shocks for a single conservation law, SIAM J. Math. Anal. 19 (1988), 1391-1408.

[21] A. Pliś, Characteristics of nonlinear partial differential equations, Bull. Acad. Polon. Sci. Cl. III 2 (1954), 419-422.

[22] M. Tsuji, Formation of singularities for Hamilton-Jacobi equation II, J. Math. Kyoto Univ. 26 (1986), 299-308. 
[23] M. Tsuji, Prolongation of classical solutions and singularities of generalized solutions, Ann. Inst. H. Poincarè Anal. Non Linéaire 7 (1990), 505-523.

[24] M. Tsuji, Formation of singularities for Monge-Ampère equations, Bull. Sci. Math. 119 (1995), 433-457.

[25] H. Whitney, On singularities of mappings of Euclidean spaces, I, Ann. of Math. (2) 62 (1955), 374-410. 